\title{
Mutational spectrum of SMPD1 gene in Pakistani Niemann-Pick disease patients
}

\author{
Huma Arshad Cheema', Iqra Ghulam Rasool'2, \\ Muhammad Nadeem Anjum ${ }^{3}$, Muhammad Yasir Zahoor ${ }^{4}$
}

\begin{abstract}
Objective: Genetic variation analysis of rare autosomal recessive Niemann-Pick disease (NPD) Pakistani patients.

Methods: We sequenced the SMPD1 gene including its all coding and flanking regions in seven unrelated sporadic patients suffering from Niemann-Pick disease through targeted exome sequencing. Genetic variants mapping and their protein predictions were evaluated using different bioinformatics tools and clinical phenotypes were correlated. The study was conducted from January 2018 to March 2019 at The Children's Hospital Lahore.

Results: We have mapped five different mutations in SMPD1 gene of enrolled patients with a novel homozygous missense variant (c.1718G $>C$ ) (p. Trp573Ser) in one patient. A missense mutation (c.1267C $>$ T) (p.His423Tyr) has been identified in three unrelated patients. A nonsense mutation (c.1327C >T) (p.Arg443Term) and one missense mutation (c.1493G >A) (p.Arg498His) mapped in one patient each. A compound heterozygous mutation has been mapped in one patient (c.740G>A) (p.Gly247Asp); (c.1493G>A) (p.Arg498His). Pathogenic effect of novel variant has been predicted through in-silico analysis and has not been reported in general overall population in the globe.

Conclusion: This is the first report of genetic demographic assessment of Niemann-Pick disease in Pakistan. The mapped mutations would be helpful to build a disease variants algorithm of Pakistani population. This will be used for determining disease clinical magnitude along with provision of genetic screening services in affected families.
\end{abstract}

KEYWORDS: Acid sphingomyelinase, Mutations, Niemann-Pick disease, SMPD1.

doi: https://doi.org/10.12669/pjms.36.3.467

How to cite this:

Cheema HA, Rasool IG, Anjum MN, Zahoor MY. Mutational spectrum of SMPD1 gene in Pakistani Niemann-Pick disease patients. Pak J Med Sci. 2020;36(3):479-484. doi: https://doi.org/10.12669/pjms.36.3.467

This is an Open Access article distributed under the terms of the Creative Commons Attribution License (http://creativecommons.org/licenses/by/3.0), which permits unrestricted use, distribution, and reproduction in any medium, provided the original work is properly cited.

1. Dr. Huma Arshad Cheema, MBBS, MCPS, DPGN.

2. Iqra Ghulam Rasool, M.Phil.

3. Dr. Muhammad Nadeem Anjum, MBBS, FCPS.

4. Dr. Muhammad Yasir Zahoor, PhD.

1,3: Department of Pediatric Gastroenterology and Hepatology, The Children's Hospital \& The Institute for Child Health, Lahore, Pakistan.

2,4: Institute of Biochemistry \& Biotechnology,

University of Veterinary \& Animal Sciences, Lahore, Pakistan.

Correspondence:

Dr. Muhammad Yasir Zahoor, PhD.

Associate Professor, Molecular Biology \& Forensic Laboratory, Institute of Biochemistry \& Biotechnology,

University of Veterinary \& Animal Sciences, Lahore, Pakistan. Email: yasir.zahoor@uvas.edu.pk

* Received for Publication:

* $1^{\text {st }}$ Revision Received:

* $2^{\text {nd }}$ Revision Received:

* Final Revision Accepted:
March 8, 2019

September 16, 2019

December 12, 2019

December 20, 2019

\section{INTRODUCTION}

Niemann-Pick disease (NPD) OMIM \# 607608; is caused by the deficiency or inactivity of acid sphingomyelinase enzyme (ASM) EC 3.1.4.12, encoded by SMPD1 gene. The disease is associated with mutations in ASM encoding gene SMPD1, resulting in sphingomyelin accumulation in reticuloendothelial cells and hepatocytes. ${ }^{1}$ Clinically, NPD patients usually present with enlargement of body organs such as liver and spleen (hepatosplenomegaly), fail to weight gaining and growth in a usual way (failure to thrive), progressive loss of mental abilities, loss of muscle movement, eye abnormality called a cherryred spot and recurrent lung infections. NPD can 
be classified into two common types: NPD type A and NPD type B, based on the onset of the disease, severity of symptoms and type of organs affected both caused by variation in SMPD1 gene. The NPD types A and B has been reported for affecting 1 in 250,000 individuals.,3 NPD type A affects in early infancy with organomegaly and then a neurodegenerative course manifested around three months of age leading to demise by three years while, with NPD type B patients often survive into adulthood correlate with hepatosplenomegaly and respiratory complications and have less or no CNS involvement. ${ }^{4,5}$

Acid sphingomyelinase is a lipid hydrolase that catalyzes sphingomyelin (SM) to phosphorylcholine and ceramide. Breakdown of SM is carried out by acid sphingomyelinases (ASMases), which cut the phosphodiester bond of SM producing the phosphorylcholine head group to generate ceramide. Deficiency or inactivity of ASMase leads to accumulation of SM resulting in Niemann-Pick disease (OMIM; NPD-A: 257200, NPD-B: 607616). ${ }^{2,3}$

The SMPD1 gene is positioned on chromosome 11p15.4-p15.1 and containing 6 exons spans approximately $\sim 6$ kilobases and encodes a protein of 631 amino acids. ${ }^{6}$ At present more than 133 missense and nonsense mutations have been reported worldwide in SMPD1 gene related to Niemann-Pick disease spreading coding domains of the gene (Human Gene Mutation Database accessed on 11-02-2019; http://www.hgmd.cf.ac. uk/ac/all.php).

Autosomal recessive disorders are present more frequently in Pakistani populations due to high rate of consanguinity. Almost $65 \%$ of the marriages are consanguineous in Pakistan among these first cousins' marriages accounts more than $74 \%{ }^{7}$ We have performed genetic investigation for causative variants of ND disease and mapped five mutations in SMPD1 gene among patients from segregating the NPD disease and have consanguineous unions.

\section{METHODS}

Enrollment of Patients: Total seven sporadic patients having Niemann-Pick disease (named PKNP 1-7) were enrolled with informed consent for genetic evaluations through collaboration of the Children Hospital Lahore. The patients were enrolled based on their clinical presentation, medical history and biochemical findings. Clinical data includes onset of the disease, hepatosplenomegaly, progressive neurodegenerative involvement, failure to thrive along with presence of reduced acid sphingomyelinase activity in fibroblasts, lymphoblasts or in peripheral white blood cells. Patients belong to different provinces as those coming to the Children Hospital Lahore being facilitated for cure of these disease. Blood samples were obtained for molecular analysis of the SMPD1 gene. NPC1 \& NPC2 were not screened as the patients were not manifesting related symptoms and variants mapped in SMPD1. The study was conducted from January 2018 to March 2019 at The Children's Hospital Lahore.

DNA extraction and targeted Sequencing: DNA was isolated using standard protocols from the blood samples of enrolled patients. The extracted DNA was stored under appropriate conditions for future analysis after analyzing quantity and quality using spectrophotometric analysis. To identify the underlying pathogenic mutation causing the disease, DNA of the affected probands were subjected to targeted exome sequencing of all coding exons and flanking intronic regions of SMPD1 gene (HiSeq 2500 System, Illumina, Archimed Life, Vienna, Austria). Data were analyzed using various bioinformatics tools for variation analysis and protein prediction.

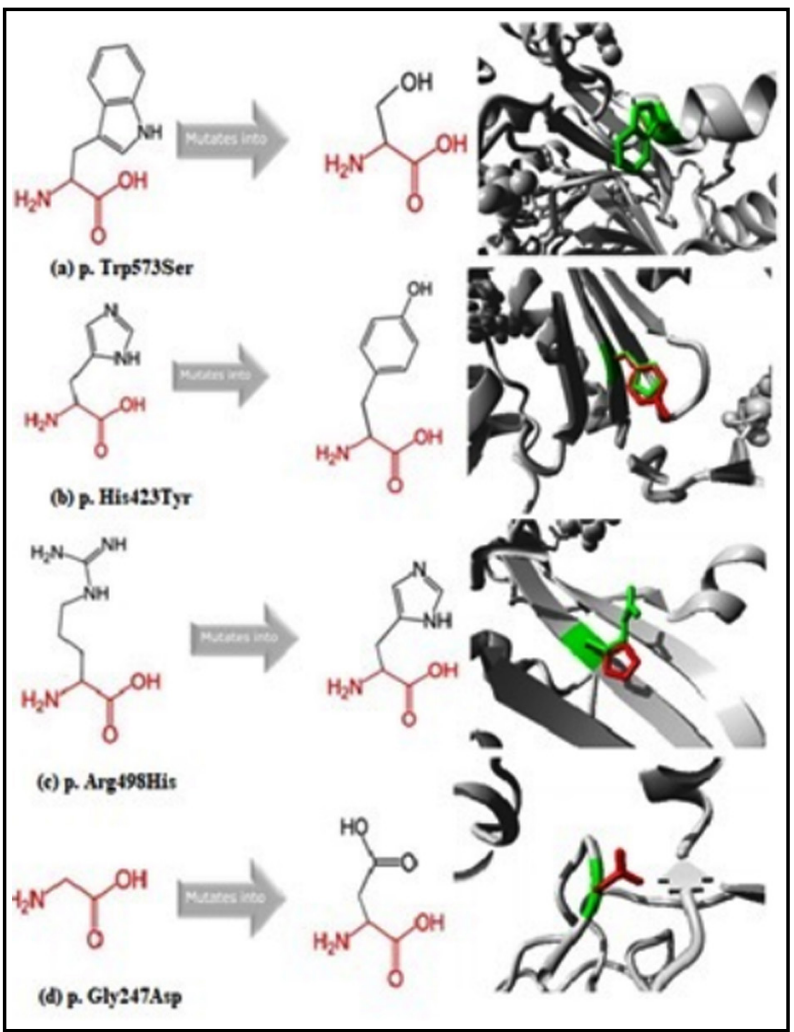

Fig.1: Description of wild type and mutant amino acids and structural changes (green color depicts wild type whereas red color showed mutant type residue). 


\section{RESULTS}

We have identified five different disease causing mutations of SMPD1 gene in Niemann-Pick disease unrelated patients from consanguineous families. A novel variant (c.1718G $>$ C) (p.Trp573Ser) has been identified in one patient in homozygous form. A missense mutation (c.1267C $>\mathrm{T}$ ) (p.His423Tyr) identified in three different patients in recessive form. A nonsense mutation (c.1327C>T) (p.Arg443Term) has been illustrated in one patient. Another homozygous missense variant (c.1493G>A) (p.Arg498His) has been mapped in another patient. While a compound heterozygous mutation has been mapped in one patient (c.740G>A) (p. Gly247Asp); (c.1493G>A) (p. Arg498His). The brief medical history of the patients has been described in Table-I. The structural descriptions of variants have been described in Fig.1.

\section{DISCUSSION}

Acid sphingomyelinase (ASM) is an enzyme that hydrolyze lipids and its deficiency or low activity lead to Niemann-Pick disease types A and B. ${ }^{8}$ ASM catalyzes the hydrolytic cleavage of sphingomyelin in lysosomes, producing phosphocholine and ceramide. ${ }^{9}$ Enzyme inactivity cause the progressive accumulation of sphingomyelin and other lipids in target tissues, and is clinically also termed as ASM deficiency disease (ASMD) responsible for the clinical spectrum of NPD-A or NPD-B metabolic phenotypes. ${ }^{10}$ The pattern of inheritance of ASMD is autosomal recessive, and associated with variants of SMPD1. Carrier frequency of SMPD1 gene variants varies among populations and some of them restricted to certain families and distinct groups. ${ }^{9-12}$ The inborn error of metabolism had mostly recessive pattern of inheritance and have increased likelihood to prevail in consanguineous population. ${ }^{13-15}$ Here, we demonstrate that the genetic variants of SMPD1 gene is Pakistani NPD patients along with carriers influences on the disease phenotype.

c. 1718G>C mutation: We have detected a novel homozygous variant c.1718G>C (p. Trp573Ser) in one patient (PKNP1). The patient was presented at age of one year with progressive abdominal distension and vomiting and belongs to Mianwaali, Punjab Pakistan. The novel c.1718G $>$ C variant produces a replacement of Tryptophan (Try) with Serine (Ser) at position 573. Wild type residue (tryptophan) contains positive charge while mutated residue (serine) contain hydroxyl group which indicates the difference in their hydrophobicity and size (Fig.1a). ${ }^{16}$ The position of amino acid is located in the core of the protein which predicted that mutated residue will cause loss of hydrophobic interaction and places an unoccupied space in the core of protein. ${ }^{17}$ The loss of interaction between these domains by the mutation reduces protein catalytic activity. A different variant at 573 position with termination codon has been reported in Italian patient. ${ }^{18}$ This novel variant was not found in Human Gene Mutation Database (http://www.hgmd.cf.ac.uk/ac/index.php) accessed on 12-02-2019, supporting the notion that this is not a reported variant previously. Furthermore, bioinformatics tools predicted this variant (p. W573S) as "damaging" the protein in structure and function as well.

c.1267C > T mutation: The c.1267C > T (p. His423Tyr) mutation was identified in three unrelated probands/patients (named PKNP4, 6, 7). PKNP4 is a 13 months old child, belongs to KPK province, Pakistan, presented with abdominal distention (liver: $7 \mathrm{~cm}$; spleen: $12 \mathrm{~cm}$ ), loose motion and worse chest infection. PKNP6 is three years old baby girl migrated from Kabul, Afghanistan in KPK. She had mild to moderate developmental delay, abdominal distention (liver: $13 \mathrm{~cm}$; spleen: $20 \mathrm{~cm}$ ) and history of PCV (packed red blood cells) transfusion. She is also having recurrent respiratory tract infections. PKNP7 is a 21 months old child, belongs to Interior Sindh, having moderate developmental delay, progressive pallor and abdominal distension (liver: $6 \mathrm{~cm}$; spleen: $14 \mathrm{~cm}$ ) since the age six months. She was transfused PCV twice during infancy and has history of recurrent chest infections and one-time admission due to RTI (Respiratory tract Infections). General physical examination showed that she is pale, underweight and stunted.

Mutation c.1267C $>\mathrm{T}$ depicted that wild type residue (Histidine) has less hydrophobicity than mutated residue (Tyrosine) and is larger in size than wild type. The difference in size of mutant residue and wild-type displaces the position so that the new residue is not at a place to make the same hydrogen bond as the wild-type residue fixes normally. The wild-type residue was buried in the core of the protein and new variant mutation disrupts correct folding of protein through loss of hydrogen bonds in the core of the protein (Fig.1b). Furthermore, various bioinformatics tools also have been predicting a damaging mutation. The 
Huma Arshad Cheema et al.

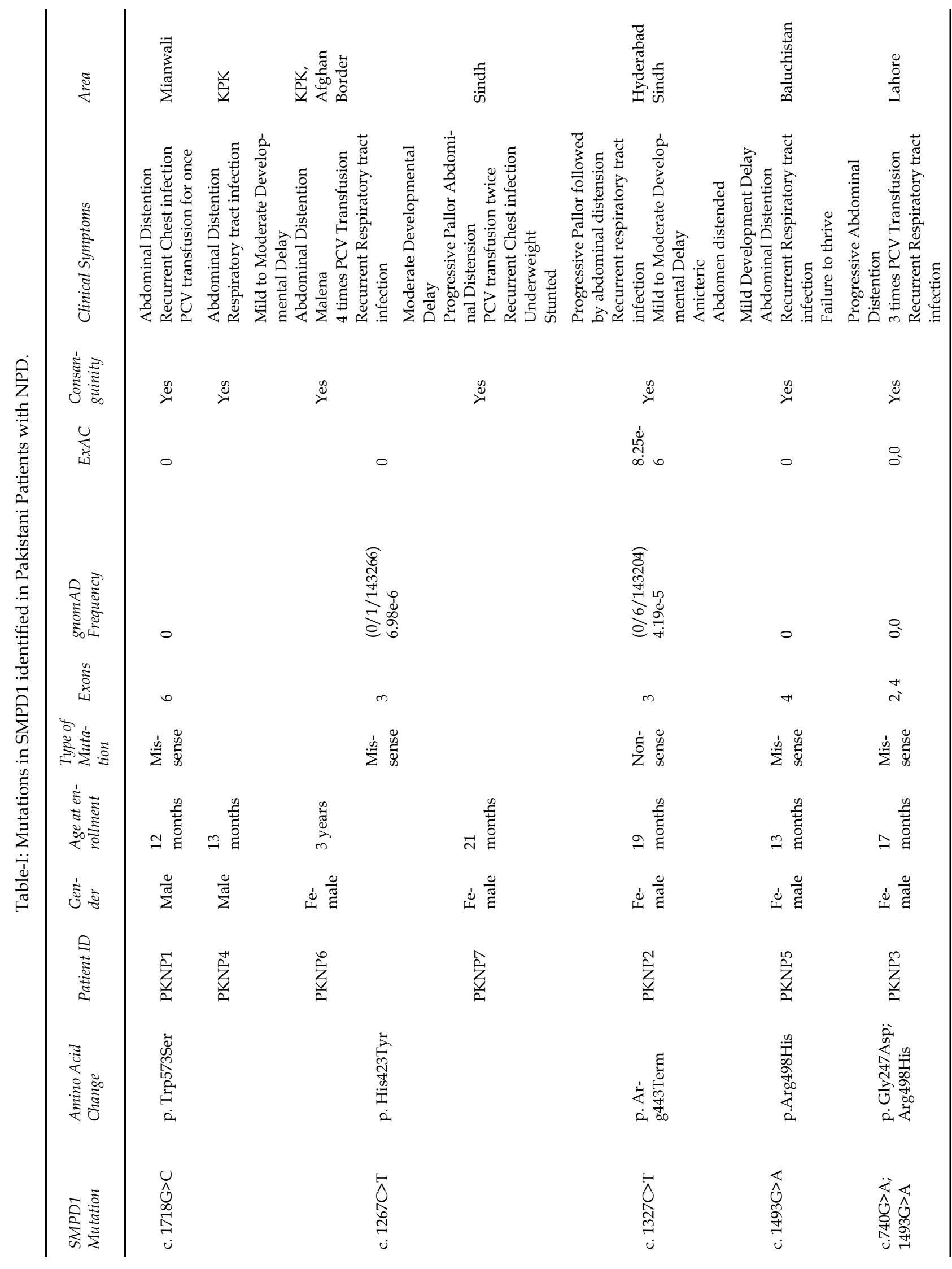

Pak J Med Sci $\quad$ March - April 2020 Vol. 36 No. $3 \quad$ www.pjms.org.pk 482 
c.1267C > T (p. His423Tyr) mutation was reported in Saudi Arabian patients, which accounts for the $\sim 85 \%$ of the disease allele in those patients. ${ }^{19}$ Here, we report this mutation in three unrelated Pakistani patients and can be predicted that this disease allele may have more penetration in our population and can be among major screening variants in affected families.

c. 1327C $>$ T mutation: A mutation with termination codon (c. 1327C >T) (p. Arg443Term) was detected in one patient (PKNP2). PKNP2 is a 19 months old child from Hyderabad, presented with progressive pallor followed by abdominal distension (liver: $5 \mathrm{~cm}$; spleen: $14 \mathrm{~cm}$ ) since the age of seven months. He has mild to moderate developmental delay and has history of recurrent respiratory tract infections. On examination, he was found pale and anicteric. No ascites was observed.

Mutation (c. 1327C > T) showed that the wild type residue (Arginine) was replaced by stop codon. This variant forms a premature termination codon at position 443; Arg443Term. This mutation leads to the formation of non-functional protein. The (c.1327C > T) (p.Arg443Term) mutation has been reported in Asian Indian descent of Niemann-Pick disease. ${ }^{20}$

c. 1493G $>A$ mutation: The (c.1493G $>$ A) (p.Arg498His) missense mutation was mapped in one patient (PKNP5) in homozygous form and heterozygous form in one patient PKNP3. PKNP5 is a two and half years old child who belongs to Baluchistan, Pakistan. She was suffering from mild development delay and showed progressive abdominal distention since age one year. Other symptoms include recurrent respiratory tract infection and failure to thrive.

Mutation (c. 1493G>A) showed that the wild-type residue (Arginine) carries positive charge while the mutant residue (Histidine) has a neutral charge and is smaller in size. Arginine is involved in salt bridge formation with aspartic acid at position 463 so the difference in charge will disturb the ionic interaction made by the arginine (Fig.1c). Mutant residue is located on a significant conserved domain and such variants have highly damaging possessions. The (c. 1493G>A) mutation has been reported in Italian population. ${ }^{18}$

Compound heterozygous c.740G $>A ; 1493 G>A$ mutation: One mutations (c.740G>A); was mapped in one patient (PKNP3) in compound heterozygous form with another heterozygous variant (c.1493G>A). PKNP3 is a 17 months old child from Lahore, Pakistan, presented with progressive abdominal distention since age seven months and having history of PCV transfusion. Other symptoms include recurrent respiratory tract infections.

Mutation (c.740G>A) result in replacement of Glycine at position 247 to Aspartic acid (p. Gly247Asp). The wild type residue (Glycine) is neutral and mutant type residue (Aspartic acid) carries negative charge (Fig.1d). The size of mutant type was bigger than wild type. Due to size and charge difference, the mutant residue has not an accurate position to make a bond with other residues. This mutation (c.740G>A); (p. Gly247Asp) has been reported in two Hungarian patients. ${ }^{21}$ In second variant (c.1493G>A); (p. Arg498His), the wild-type residue (Arginine) carries positive charge while the mutant variant (Histidine) has a neutral charge and is smaller than arginine as discussed in previous segment. The (c. 1493G>A) mutation has been reported in Italian population. ${ }^{18}$ The inherited disorders in children including the lysosomal storage disorder in Pakistan need to be focused for molecular studies and the trend has been increasing as it will provide child health care. ${ }^{22-25}$

\section{CONCLUSION}

This is the first report for mutation spectrum of SMPD1 gene in Pakistan contributing to development in genomic variants algorithm of NPD in indigenous population. Molecular analysis for NPD would be helpful for early and accurate diagnosis leading to a proper disease management.

Grant Support: Partially supported by HEC, Pakistan (NRPU/6948).

Conflict of interest $\mathcal{E}$ financial disclosures: None.

\section{REFERENCES}

1. Schuchman EH, Wasserstein MP. Types A and B NiemannPick disease. Pediatr Endocrinol Rev. 2016;13(Suppl 1):674-681.

2. Schuchman EH, Wasserstein MP. Types A and B NiemannPick disease. Best Pract Res Clin Endocrinol Metab. 2015;29(2):237-247. doi: 10.1016/j.beem.2014.10.002

3. Schuchman EH, Desnick RJ. Types A and B NiemannPick disease. Mol Genet Metab. 2017;120(1-2):27-33. doi: 10.1016/j.ymgme.2016.12.008

4. Elleder M, Cihula J. Niemann-Pick disease (variation in the sphingomyelinase deficient group): Neurovisceral phenotype (A) with an abnormally protracted clinical course and variable expression of neurological symptomatology in three siblings. Eur J Pediatr. 1983;140:323-328. doi: 10.1007/bf00442673 
5. Elleder M, Nevoral J, Spicakova V, Hyniova H, Kraus J, Krasny J, et al. A new variant of sphingomyelinase deficiency (Niemann-Pick): Visceromegaly, minimal neurological lesions and low in vivo degradation rate of sphingomyelin. J Inherit Metab Dis. 1986;9:357-366. doi:10.1007/bf01800485

6. Simonaro CM, Park JH, Eliyahu E, Shtraizent N, McGovern MM, Schuchman EH. Imprinting at the SMPD1 locus: Implications for acid sphingomyelinase-deficient NiemannPick disease. Am J Hum Genet. 2006;78:865-870. doi: 10.1086/503750

7. Bhinder MA, Sadia H, Mahmood N, Qasim M Hussain Z, Rashid MM, et al. Consanguinity: A blessing or menace at population level? Ann Hum Genet. 2019;83(4)214-219. doi: 10.1111/ahg.12308

8. Zampieri S, Filocamo M, Pianta A, Lualdi S, Gort L, Coll MJ, et al.SMPD1 mutation update: database and comprehensive analysis of published and novel variants. Hum Mutat. 2016;37:39-47. doi: 10.1002/humu.22923

9. McGovern MM, Dionisi-Vici C, Giugliani R, Hwu P, Lidove O, Lukacs $\mathrm{Z}$, et al. Consensus recommendation for a diagnostic guideline for acid sphingomyelinase deficiency. Genet Med. 2017;19:967-974. doi: 10.1038/gim.2017.7

10. Wasserstein MP, Aron A, Brodie SE, Simonaro C, Desnick RJ, McGovern MM. Acid sphingomyelinase deficiency: Prevalence and characterization of an intermediate phenotype of Niemann-Pick disease. J Pediatr. 2006;149(4):554-559. doi: 10.1016/j.jpeds.2006.06.034

11. Acuna M, Martinez P, Moraga C, He X, Moraga M, Hunter B, et al. Epidemiological, clinical and biochemical characterization of the p.(Ala359Asp) SMPD1variant causing Niemann-Pick disease type B. Eur J Hum Genet. 2016;24:208-213. doi: 10.1038/ejhg.2015.89

12. Burton BK, Charrow J, Hoganson GE, Waggoner D, Tinkle B, Braddock SR, et al. Newborn screening for lysosomal storage disorders in Illinois: the initial 15-month experience. J Pediatr. 2017;190:130-135. doi: 10.1016/j.jpeds.2017.06.048

13. Zahoor MY, Cheema HA, IjazS, FayyazZ. Genetic analysis of tyrosinemia type 1 and fructose- 1,6 bisphosphatase deficiency affected Pakistani cohorts. Fetal Pediatr Pathol. 2019. doi: 10.1080/15513815.2019.1672224 [Epub ahead of print]

14. Ijaz S, Zahoor MY, Imran M, Ramzan K, Bhinder MA, Iqbal $M$, et al. Genetic analysis of FBPase deficiency in nine consanguineous Pakistani families. J Pediatr Endocrinol Metab. 2017;30(11):1203-1210. doi:10.1515/jpem-2017-0188

15. Zahoor MY, Cheema HA, Ijaz S, Anjum MN, Ramzan K, Bhinder MA. Mapping of IDUA gene variants in Pakistani patients with mucopolysaccharidosis type 1 . J Pediatr Endocrinol Metab. 2019;32(11):1221-1227. doi: 10.1515/ jpem-2019-0188

16. Gorelik A, Illes K, Heinz LX, Superti-Furga G, Nagar B. Crystal structure of mammalian acid sphingomyelinase. Nat Commun. 2016;7:12196. doi: 10.1038/ncomms12196
17. Kelley LA, Mezulis S, Yates CM, Wass MN, Sternberg MJ. The Phyre2 web portal for protein modeling, prediction and analysis. Nat Protoc. 2015;10:845-858. doi: 10.1038/ nprot.2015.053

18. Ricci V, Stroppiano M, Corsolini F, Rocco MD, Parenti G, Regis S, et al. Screening of 25 Italian Patients with NiemannPick A Reveals Fourteen New Mutations, One Common and Thirteen Private, in SMPD1. Hum Mutat. 2004;24(1):105. doi: 10.1002/humu.9258

19. Simonaro CM, Desnick RJ, McGovern MM, Wasserstein $\mathrm{MP}$, Schuchman EH. The demographics and distribution of type $\mathrm{b}$ niemann-pick disease: Novel mutations lead to new genotype/phenotype correlations. Am J Hum Genet. 2002;71(6):1413-1419. doi: 10.1086/345074

20. Schuchman EH. Two new mutations in the acid sphingomyelinase gene causing type a Niemann-Pick Disease: N389T and R44lX. Hum Muta. 1995;6:352-354. doi: 10.1002/humu.1380060412

21. Toth B, Erdos M, Szekely A, Ritli L, Bagossi P, Sumegi $\mathrm{J}$, et al. Molecular Genetic Characterization of Novel Sphingomyelin Phosphodiesterase 1 Mutations Causing Niemann-Pick Disease. JIMD Rep. 2012;3:125-129. doi: 10.1007/8904_2011_80

22. Cheema HA, Malik HS, Parkash A, Fayyaz Z. Spectrum of inherited metabolic disorders in Pakistani children presenting at a tertiary care centre. J Coll Physician Surg Pak. 2016;26(6):498-502.

23. Ali O, Hafeez A, Ijaz A, Lodhi MA, Nawaz MA, Ahmed Z. A Novel Clinico-Biochemical Score for Screening of Inherited Metabolic Diseases in Children. J Coll Physician Surg Pak. 2018;28(11):853-857.

24. Afroze B, Brown N. Ethical issues in managing Lysosomal storage disorders in children in low and middle income countries. Pak J Med Sci. 2017;33(4):1036-1041. doi: $10.12669 /$ pjms.334.12975

25. Shahid MNS, Zahid D, Hassan J, Ansari S, Shamsi T. Alpha thalassemia deletions found in suspected cases of beta thalassemia major in Pakistani population. Pak J Med Sci. 2017;33(2):411-416. doi: 10.12669/pjms.332.11834

\section{Authors' Contribution:}

HAC \& MYZ executed work and are responsible and accountable for the accuracy or integrity of the work.

MNA did data collection and patients enrollment. IGR \& MYZ performed data analysis and manuscript writing. 